\section{Positive solutions for $m$-point boundary value problem}

\author{
Hua Su${ }^{a, b, *}$, Xinjun Wang ${ }^{a}$ \\ ${ }^{a}$ School of economics, Shandong University,250014, Jinan, China. \\ ${ }^{b}$ School of Mathematics and Quantitative Economics, Shandong University of Finance and Economics, 250014, Jinan, China.
}

Communicated by Y. J. Cho

\begin{abstract}
In this paper, we study the existence of positive solutions for the following nonlinear $m$-point boundary value problem for an increasing homeomorphism and homomorphism with sign changing nonlinearity:

$$
\left\{\begin{array}{l}
\left(\phi\left(u^{\prime}\right)\right)^{\prime}+a(t) f(t, u(t))=0, \quad 0<t<1, \\
u^{\prime}(0)=\sum_{i=1}^{m-2} a_{i} u^{\prime}\left(\xi_{i}\right), u(1)=\sum_{i=1}^{k} b_{i} u\left(\xi_{i}\right)-\sum_{i=k+1}^{s} b_{i} u\left(\xi_{i}\right)-\sum_{i=s+1}^{m-2} b_{i} u^{\prime}\left(\xi_{i}\right),
\end{array}\right.
$$

where $\phi: R \longrightarrow R$ is an increasing homeomorphism and homomorphism and $\phi(0)=0$. The nonlinear term $f$ may change sign. As an application, an example to demonstrate our results has given. The conclusions in this paper essentially extend and improve the known results. (c)2016 All rights reserved.
\end{abstract}

Keywords: $m$-point boundary value problem, positive solutions, fixed-point theorem.

$2010 M S C: 34 \mathrm{~B} 16$.

\section{Introduction}

In this paper, we study the existence of positive solutions of the following nonlinear $m$-point boundary value problem with sign changing nonlinearity:

$$
\left\{\begin{array}{l}
\left(\phi\left(u^{\prime}\right)\right)^{\prime}+a(t) f(t, u(t))=0, \quad 0<t<1, \\
u^{\prime}(0)=\sum_{i=1}^{m-2} a_{i} u^{\prime}\left(\xi_{i}\right), u(1)=\sum_{i=1}^{k} b_{i} u\left(\xi_{i}\right)-\sum_{i=k+1}^{s} b_{i} u\left(\xi_{i}\right)-\sum_{i=s+1}^{m-2} b_{i} u^{\prime}\left(\xi_{i}\right),
\end{array}\right.
$$

where $\phi: R \longrightarrow R$ is an increasing homeomorphism and homomorphism and $\phi(0)=0 ; \xi_{i} \in(0,1)$ with

\footnotetext{
${ }^{*}$ Corresponding author

Email addresses: jnsuhua@163.com (Hua Su), showme1@yeah.net (Xinjun Wang)
} 
$0<\xi_{1}<\xi_{2}<\cdots<\xi_{m-2}<1$ and $a_{i}, b_{i}, a$ and $f$ satisfy

$\left(H_{1}\right) \quad a_{i}, b_{i} \in[0,+\infty), 0<\sum_{i=1}^{k} b_{i}-\sum_{i=k+1}^{s} b_{i}<1,0<\sum_{i=1}^{m-2} a_{i}<1$

$\left(H_{2}\right) \quad a(t):(0,1) \rightarrow[0,+\infty)$ does not vanish identically on any subinterval of $[0,1]$ and satisfies

$$
0<\int_{0}^{1} a(t) \mathrm{d} t<\infty
$$

$\left(H_{3}\right) \quad f \in C([0,1] \times[0,+\infty),(-\infty,+\infty)), f(t, 0) \geq 0$ and $f(t, 0) \neq 0$.

Definition 1.1. A projection $\phi: R \longrightarrow R$ is called an increasing homeomorphism and homomorphism, if the following conditions are satisfied:

(i) if $x \leq y$, then $\phi(x) \leq \phi(y) \forall x, y \in R$;

(ii) $\phi$ is a continuous bijection and its inverse mapping is also continuous;

(iii) $\phi(x y)=\phi(x) \phi(y) \forall x, y \in R$.

The study of multi-point boundary value problems for linear second-order ordinary differential equations was initiated by Il'in and Moiseev [3, 4]. Motivated by the study of [3, 4], Gupta [2] studied certain threepoint boundary value problems for nonlinear ordinary differential equations. Since then, more general nonlinear multi-point boundary value problems have been studied by several authors. We refer the reader to [5, 8, 11, 12, 14, 15, 16, 17, 18, 19] and [20] for some references along this line. Multi-point boundary value problems describe many phenomena in the applied mathematical sciences. For example, the vibrations of a guy wire of a uniform cross-section and composed of $\mathrm{N}$ parts of different densities can be set up as a multi-point boundary value problems (see Moshinsky [7]); many problems in the theory of elastic stability can be handle by the method of multi-point boundary value problems(see Timoshenko [13]).

In 2001, Ma [5] studied $m$-point boundary value problem (BVP)

$$
\left\{\begin{array}{l}
u^{\prime \prime}(t)+h(t) f(u)=0, \quad 0 \leq t \leq 1, \\
u(0)=0, \quad u(1)=\sum_{i=1}^{m-2} \alpha_{i} u^{\prime}\left(\xi_{i}\right),
\end{array}\right.
$$

where $\alpha_{i}>0(i=1,2, \cdots, m-2), \quad \sum_{i=1}^{m-2} \alpha_{i}<1,0<\xi_{1}<\xi_{2}<\cdots<\xi_{m-2}<1, f \in C([0,+\infty),[0,+\infty))$ and $h \in C([0,1],[0,+\infty))$. Author established the existence of positive solutions theorems under the condition that $f$ is either superlinear or sublinear.

Recently, Ma et al. [6] used the monotone iterative technique in cones to prove the existence of at least one positive solutions for m-point boundary value problem (BVP)

$$
\left\{\begin{array}{l}
\left(\phi_{p}\left(u^{\prime}\right)\right)^{\prime}+a(t) f(t, u(t))=0, \quad 0<t<1, \\
u^{\prime}(0)=\sum_{i=1}^{m-2} a_{i} u^{\prime}\left(\xi_{i}\right), \quad u(1)=\sum_{i=1}^{m-2} b_{i} u\left(\xi_{i}\right),
\end{array}\right.
$$

where $0<\sum_{i=1}^{m-2} b_{i}<1, \quad 0<\sum_{i=1}^{m-2} a_{i}<1, \quad 0<\xi_{1}<\xi_{2}<\cdots<\xi_{m-2}<1, a(t) \in L^{1}[0,1]$ and $f \in C([0,1] \times[0,+\infty),[0,+\infty))$. The main tool is the monotone iterative technique.

In [15], $\mathrm{Xu}$ studied the existence of positive solutions for (1.1). However, in all the above mentioned papers, the authors discuss about the boundary value problem (BVP) under the key conditions that nonlinear term is positive continuous function. Motivated by the results mentioned above, in this paper we study the existence of positive solutions of $m$-point boundary value problem (1.1) for an increasing homeomorphism and homomorphism with sign changing nonlinearity. We generalize the results in [5, 8, 11, 12, 14.

By a positive solution of BVP (1.1), we understand a function $u$ which is positive on $(0,1)$ and satisfies the differential equations as well as the boundary conditions in BVP (1.1). 


\section{Preliminaries and Lemmas}

In this section, we present some lemmas which are important to our main results.

Lemma 2.1. Let $\left(H_{1}\right)$ and $\left(H_{2}\right)$ hold. Then for $u \in C^{+}[0,1]$, the problem

$$
\left\{\begin{array}{l}
\left(\phi\left(u^{\prime}\right)\right)^{\prime}+a(t) f(t, u(t))=0, \quad 0<t<1, \\
u^{\prime}(0)=\sum_{i=1}^{m-2} a_{i} u^{\prime}\left(\xi_{i}\right), u(1)=\sum_{i=1}^{k} b_{i} u\left(\xi_{i}\right)-\sum_{i=k+1}^{s} b_{i} u\left(\xi_{i}\right)-\sum_{i=s+1}^{m-2} b_{i} u^{\prime}\left(\xi_{i}\right),
\end{array}\right.
$$

has a unique solution $u(t)$ if and only if $u(t)$ can be express as the following equation

$$
u(t)=-\int_{t}^{1} \omega_{f}(s) d s+B
$$

where $A, B$ satisfy

$$
\begin{gathered}
\phi^{-1}(A)=\sum_{i=1}^{m-2} a_{i} \phi^{-1}\left(A-\int_{0}^{\xi_{i}} a(s) f(s, u(s)) d s\right) \\
B=-\frac{1}{1-\sum_{i=1}^{k} b_{i}+\sum_{i=k+1}^{s} b_{i}}\left[\sum_{i=1}^{k} b_{i} \int_{\xi_{i}}^{1} \omega_{f}(s) d s-\sum_{i=k+1}^{s} b_{i} \int_{\xi_{i}}^{1} \omega_{f}(s) d s\right. \\
\left.+\sum_{i=s+1}^{m-2} b_{i} \phi^{-1}\left(A-\int_{0}^{\xi_{i}} a(s) f(s, u(s)) d s\right)\right],
\end{gathered}
$$

where

$$
\omega_{f}(s)=\phi^{-1}\left(-\int_{0}^{s} a(r) f(\tau, u(r)) d r+A\right)
$$

Define $l=\frac{\phi\left(\sum_{i=1}^{m-2} a_{i}\right)}{1-\phi\left(\sum_{i=1}^{m-2} a_{i}\right)} \in(0,1)$, then there exists a unique $A \in\left[-l \int_{0}^{1} a(s) f(t, u(s)) d s, 0\right]$ satisfying (2.2).

Proof. The method of the proof is similar to Lemma 2.1 in [7], we omit the details.

Lemma 2.2. Let $\left(H_{1}\right)$ and $\left(H_{2}\right)$ hold. If $u \in C^{+}[0,1]$, the unique solution of the problem (2.1) satisfies

$$
u(t) \geq 0, t \in[0,1] .
$$

Proof. The method of the proof is similar to Lemma 2.2 in [15], we omit the details.

Lemma 2.3. Let $\left(H_{1}\right)$ and $\left(H_{2}\right)$ hold. If $u \in C^{+}[0,1]$, the unique solution of the problem (2.1) satisfies

$$
\inf _{t \in[0,1]} u(t) \geq \gamma\|u\|
$$

where

$$
\gamma=\frac{\left(\sum_{i=1}^{k} b_{i}-\sum_{i=k+1}^{s} b_{i}\right)\left(1-\xi_{k}\right)}{1-\sum_{i=1}^{k} b_{i} \xi_{k}+\sum_{i=k+1}^{s} b_{i} \xi_{k}} \in(0,1),\|u\|=\max _{t \in[0,1]}|u(t)|
$$


Proof. The method of the proof is similar to Lemma 2.3 in [15], we omit the details.

Lemma 2.4 ([1]). Let $K$ be a cone in a Banach space $X$. Let $D$ be an open bounded subset of $X$ with $D_{K}=D \cap K \neq \phi$ and $\overline{D_{K}} \neq K$. Assume that $A: \overline{D_{K}} \longrightarrow K$ is a compact map such that $x \neq A K$ for $x \in \partial D_{K}$. Then the following results hold:

(1) if $\|A x\| \leq\|x\|$ for all $x \in \partial D_{K}$, then $i\left(A, D_{K}, K\right)=1$;

(2) if there exists $x_{0} \in K \backslash\{\theta\}$ such that $x \neq A x+\lambda x_{0}$, for all $x \in \partial D_{K}$ and all $x>0$, then $i\left(A, D_{K}, K\right)=$ 0 ;

(3) let $U$ be open in $X$ such that $\bar{U} \subset D_{K}$. If $i\left(A, D_{K}, K\right)=1$ and $i\left(A, D_{K}, K\right)=0$,

then $A$ has a fixed point in $D_{K} \backslash \bar{U}_{K}$. The same results holds, if $i\left(A, D_{K}, K\right)=0$ and $i\left(A, D_{K}, K\right)=1$.

Let $E=C[0,1]$, then $E$ is Banach space, with respect to the norm $\|u\|=\sup _{t \in[0,1]}|u(t)|$. Denote

$$
K=\left\{u \mid u \in C[0,1], u(t) \geq 0, \inf _{t \in[0,1]} u(t) \geq \gamma\|u\|\right\},
$$

where $\gamma$ is the same as in Lemma 2.3. It is obvious that $K$ is a cone in $C[0,1]$. We define $\varphi(t)=$ $\min \{t, 1-t\}, t \in(0,1)$ and

$$
\begin{aligned}
K_{\rho} & =\{u(t) \in K:\|u\|<\rho\} \\
K_{\rho}^{*} & =\{u(t) \in K: \rho \varphi(t)<u(t)<\rho\} \\
\Omega_{\rho} & =\left\{u(t) \in K: \min _{\xi_{m-2} \leq t \leq 1} u(t)<\gamma \rho\right\} \\
& =\left\{u(t) \in E: u \geq 0, \gamma\|u\| \leq \min _{\xi_{m-2} \leq t \leq 1} u(t)<\gamma \rho\right\} .
\end{aligned}
$$

Lemma 2.5 ([7]). $\Omega_{\rho}$ defined above has the following properties:

(a) $K_{\gamma \rho} \subset \Omega_{\rho} \subset K_{\rho}$

(b) $\Omega_{\rho}$ is open relative to $K$;

(c) $X \in \partial \Omega_{\rho}$ if and only if $\min _{\xi_{m-2} \leq t \leq 1} x(t)=\gamma \rho$

(d) if $x \in \partial \Omega_{\rho}$, then $\gamma \rho \leq x(t) \leq \rho$ for $t \in\left[\xi_{m-2}, 1\right]$.

Now, for the convenience, we introduce the following notations,

$$
\begin{aligned}
& f_{\gamma \rho}^{\rho}=\min \left\{\min _{\xi_{m-2} \leq t \leq 1} \frac{f(t, u)}{\phi(\rho)}: u \in[\gamma \rho, \rho]\right\}, \\
& f_{0}^{\rho}=\max \left\{\max _{0 \leq t \leq 1} \frac{f(t, u)}{\phi(\rho)}: u \in[0, \rho]\right\}, \\
& f_{\varphi(t) \rho}^{\rho}=\max \left\{\max _{0 \leq t \leq 1} \frac{f(t, u)}{\phi(\rho)}: u \in[\varphi(t) \rho, \rho]\right\}, \\
& f^{\alpha}=\lim _{u \rightarrow \alpha} \sup \max _{0 \leq t \leq 1} \frac{f(t, u)}{\phi(u)}, \\
& f_{\alpha}=\lim _{u \rightarrow \alpha} \inf \min _{\xi_{m-2} \leq t \leq 1} \frac{f(t, u)}{\phi(u)},\left(\alpha:=\infty \text { or } 0^{+}\right),
\end{aligned}
$$




$$
\begin{aligned}
& m=\left\{\frac{\left(1+\sum_{i=k+1}^{s} b_{i}+\sum_{i=s+1}^{m-2} b_{i}\right) \phi^{-1}\left((l+1) \int_{0}^{1} a(s) \mathrm{d} s\right)}{1-\sum_{i=1}^{k} b_{i}+\sum_{i=k+1}^{s} b_{i}}\right\}^{-1}, \\
& M=\left\{\frac{\sum_{i=1}^{k} b_{i}-\sum_{i=k+1}^{s} b_{i}}{1-\sum_{i=1}^{k} b_{i}+\sum_{i=k+1}^{s} b_{i}} \int_{\xi_{k}}^{1} \phi^{-1}\left(\int_{0}^{s} a(r) \mathrm{d} r\right) \mathrm{d} s\right\}^{-1},
\end{aligned}
$$

\section{Main results}

In the rest section, we also assume the conditions:

$\left(A_{1}\right)$ There exist $\rho_{1}, \rho_{2} \in(0,+\infty)$ with $\rho_{1}<\gamma \rho_{2}$ such that

(1) $f(t, u)>0, t \in[0,1], u \in\left[\rho_{1} \varphi(t),+\infty\right)$,

(2) $f_{\varphi(t) \rho_{1}}^{\rho_{1}} \leq \phi(m), f_{\gamma \rho_{2}}^{\rho_{2}} \geq \phi(M \gamma)$.

$\left(A_{2}\right)$ There exist $\rho_{1}, \rho_{2} \in(0,+\infty)$ with $\rho_{1}<\rho_{2}$ such that

(3) $f(t, u)>0, t \in[0,1], u \in\left[\min \left\{\gamma \rho_{1}, \rho_{2} \varphi(t)\right\},+\infty\right)$,

(4) $f_{\gamma \rho_{1}}^{\rho_{1}} \geq \phi(M \gamma), f_{\varphi(t) \rho_{2}}^{\rho_{2}} \leq \phi(m)$.

$\left(A_{3}\right)$ There exist $\rho_{1}, \rho_{2}, \rho_{3} \in(0,+\infty)$ with $\rho_{1}<\gamma \rho_{2}$ and $\rho_{2}<\rho_{3}$ such that

(1) $f(t, u)>0, t \in[0,1], u \in\left[\rho_{1} \varphi(t),+\infty\right)$,

(2) $f_{\varphi(t) \rho_{1}}^{\rho_{1}} \leq \phi(m), f_{\gamma \rho_{2}}^{\rho_{2}} \geq \phi(M \gamma), f_{\varphi(t) \rho_{3}}^{\rho_{3}} \leq \phi(m)$.

$\left(A_{4}\right)$ There exist $\rho_{1}, \rho_{2}, \rho_{3} \in(0,+\infty)$ with $\rho_{1}<\rho_{2}<\gamma \rho_{3}$ such that

(3) $f(t, u)>0, t \in[0,1], u \in\left[\min \left\{\gamma \rho_{1}, \rho_{2} \varphi(t)\right\},+\infty\right)$,

(4) $f_{\gamma \rho_{1}}^{\rho_{1}} \geq \phi(M \gamma), f_{\varphi(t) \rho_{2}}^{\rho_{2}} \leq \phi(m) ; f_{\gamma \rho_{3}}^{\rho_{3}} \geq \phi(M \gamma)$.

Our main results are following theorems.

Theorem 3.1. Assume that $\left(H_{1}\right),\left(H_{2}\right),\left(H_{3}\right),\left(A_{3}\right)$ hold. Then BVP 1.1 have at least three positive solutions.

Proof. Without loss of generality, we suppose that $\left(A_{3}\right)$ hold. Denote

$$
f^{*}(t, u)= \begin{cases}f(t, u), & u \geq \rho_{1} \varphi(t), \\ f\left(t, \rho_{1} \varphi(t)\right), & 0 \leq u<\rho_{1} \varphi(t),\end{cases}
$$

it is easy to check that $f^{*}(t, u) \in C([0,1] \times[0,+\infty),(0,+\infty))$.

Now define an operator $T: K \rightarrow C[0,1]$ by setting

$$
\begin{aligned}
(T u)(t)= & -\frac{1}{1-\sum_{i=1}^{k} b_{i}+\sum_{i=k+1}^{s} b_{i}}\left[\sum_{i=1}^{k} b_{i} \int_{\xi_{i}}^{1} \omega(s) \mathrm{d} s-\sum_{i=k+1}^{s} b_{i} \int_{\xi_{i}}^{1} \omega(s) \mathrm{d} s\right. \\
& \left.+\sum_{i=s+1}^{m-2} b_{i} \phi^{-1}\left(A-\int_{0}^{\xi_{i}} a(s) f^{*}(s, u(s)) \mathrm{d} s\right)\right]-\int_{t}^{1} \omega(s) \mathrm{d} s,
\end{aligned}
$$


where

$$
\omega(s)=\phi^{-1}\left(-\int_{0}^{s} a(r) f^{*}(\tau, u(r)) \mathrm{d} r+A\right) .
$$

By Lemma 2.3, we have $T(K) \subset K$. So by applying Arzela-Ascoli Theorem, we can obtain that $T(K)$ is relatively compact. In view of Lebesgue's dominated convergence theorem, it is easy to prove that $T$ is continuous. Hence, $T: K \rightarrow K$ is completely continuous.

Now, we consider the following modified BVP (1.1),

$$
\left\{\begin{array}{l}
\left(\phi\left(u^{\prime}\right)\right)^{\prime}+a(t) f^{*}(t, u(t))=0, \quad 0<t<1 \\
u^{\prime}(0)=\sum_{i=1}^{m-2} a_{i} u^{\prime}\left(\xi_{i}\right), u(1)=\sum_{i=1}^{k} b_{i} u\left(\xi_{i}\right)-\sum_{i=k+1}^{s} b_{i} u\left(\xi_{i}\right)-\sum_{i=s+1}^{m-2} b_{i} u^{\prime}\left(\xi_{i}\right) .
\end{array}\right.
$$

Obviously, BVP (3.1) has a solution $u(t)$ if and only if $u$ is a fixed point of the operator $T$. From the condition $\left(A_{3}\right)(2)$, we have

$$
f_{\varphi(t) \rho_{1}}^{* \rho_{1}} \leq \phi(m), \quad f_{\gamma \rho_{2}}^{* \rho_{2}} \geq \phi(M \gamma), \quad f_{\varphi(t) \rho_{3}}^{* \rho_{3}} \leq \phi(m) .
$$

Next, we will show that $i\left(A, K_{\rho_{1}}^{*}, K\right)=1$.

In fact, by $f_{\varphi(t) \rho_{1}}^{* \rho_{1}} \leq \phi(m)$ for all $u \in \partial K_{\rho_{1}}^{*}$, we have

$$
\begin{aligned}
(T u)(t)= & -\frac{1}{1-\sum_{i=1}^{k} b_{i}+\sum_{i=k+1}^{s} b_{i}}\left(\sum_{i=1}^{k} b_{i} \int_{\xi_{i}}^{1} \omega(s) \mathrm{d} s-\sum_{i=k+1}^{s} b_{i} \int_{\xi_{i}}^{1} \omega(s) \mathrm{d} s\right. \\
& \left.+\sum_{i=s+1}^{m-2} b_{i} \phi^{-1}\left(A-\int_{0}^{\xi_{i}} a(s) f^{*}(s, u(s)) \mathrm{d} s\right)\right)-\int_{t}^{1} \omega(s) \mathrm{d} s \\
\leq & \frac{1}{1-\sum_{i=1}^{k} b_{i}+\sum_{i=k+1}^{s} b_{i}}\left(\sum_{i=1}^{k} b_{i} \int_{0}^{1} \phi^{-1}\left((l+1) \int_{0}^{1} a(r) f^{*}(r, u(r)) \mathrm{d} r\right) \mathrm{d} s\right. \\
& \left.+\sum_{i=s+1}^{m-2} b_{i} \phi^{-1}\left((l+1) \int_{0}^{1} a(s) f^{*}(s, u(s)) \mathrm{d} s\right)\right) \\
& +\int_{0}^{1} \phi^{-1}\left((l+1) \int_{0}^{1} a(r) f^{*}(r, u(r)) \mathrm{d} r\right) \mathrm{d} s \\
\leq & \frac{\left.1+\sum_{i=k+1}^{s} b_{i}+\sum_{i=s+1}^{m-2} b_{i}\right) \phi^{-1}\left((l+1) \int_{0}^{1} a(s) \mathrm{d} s\right)}{1-\sum_{i=1}^{k} b_{i}+\sum_{i=k+1}^{s} b_{i}} \phi^{-1}\left(\phi\left(\rho_{1}\right) \phi(m)\right) \\
= & \rho_{1}=\|u\| .
\end{aligned}
$$

This implies that $\|T u\| \leq\|u\|$ for $u \in \partial K_{\rho}^{*}$. By Lemma 2.4 (1), we have

$$
i\left(A, K_{\rho_{1}}^{*}, K\right)=1 .
$$

Furthermore, we will show that $i\left(A, K_{\rho_{2}}, K\right)=1$.

Let $e(t) \equiv 1$, for $t \in[0,1]$, then $e \in \partial K_{1}$. We claim that

$$
u \neq T u+\lambda e, \quad u \in \partial \Omega_{\rho_{2}}, \quad \lambda>0 .
$$


In fact, if not, there exist $u_{0} \in \partial \Omega_{2}$ and $\lambda_{0}>0$ such that $u_{0}=T u_{0}+\lambda_{0} e$.

By $\left(A_{3}\right)$ and Lemma 2.1, we have for $t \in[0,1]$,

$$
-\int_{0}^{s} a(\tau) f^{*}(\tau, u(\tau)) d \tau+A \leq-\phi\left(\rho_{2}\right) \phi(M \gamma)\left(\int_{0}^{s} a(\tau) d \tau\right)
$$

so that

$$
-\omega(s)=\phi^{-1}\left(-\int_{0}^{s} a(\tau) f^{*}(\tau, u(\tau)) d \tau+A\right) \geq \rho_{2} M \gamma \phi^{-1}\left[\int_{0}^{s} a(\tau) d \tau\right] .
$$

Then, we have that

$$
\begin{aligned}
u_{0}(t) & =T u_{0}(t)+\lambda_{0} e(t) \\
& \geq \frac{1}{1-\sum_{i=1}^{k} b_{i}+\sum_{i=k+1}^{s} b_{i}} \sum_{i=1}^{k}\left(b_{i} \int_{\xi_{k}}^{1}(-\omega(s)) \mathrm{d} s-\sum_{i=k+1}^{s} b_{i} \int_{\xi_{k}}^{1}(-\omega(s)) \mathrm{d} s\right)+\lambda_{0} \\
& \geq \frac{\sum_{i=1}^{k} b_{i}-\sum_{i=k+1}^{s} b_{i}}{1-\sum_{i=1}^{k} b_{i}+\sum_{i=k+1}^{s} b_{i}} \rho_{2} M \gamma \int_{\xi_{k}}^{1} \phi^{-1}\left(\int_{0}^{s} a(r) \mathrm{d} r\right) \mathrm{d} s+\lambda_{0} \\
& =\gamma \rho_{2}+\lambda_{0}
\end{aligned}
$$

This implies that $\gamma \rho_{2} \geq \gamma \rho_{2}+\lambda_{0}$, which is a contradiction. Hence, by Lemma 2.4 (2), it follows that

$$
i\left(A, \Omega_{\rho_{2}}, K\right)=0 .
$$

Finally, similar to the proof of $i\left(A, K_{\rho_{1}}^{*}, K\right)=1$, we can show that $i\left(A, K_{\rho_{3}}^{*}, K\right)=1$.

By Lemma 2.5 (a) and $\rho_{1}<\gamma \rho_{2}$ and $\rho_{2}<\rho_{3}$, we have $\bar{K}_{\rho_{1}} \subset K_{\gamma \rho_{2}} \subset \Omega_{\rho_{2}} \subset K_{\rho_{2}} \subset K_{\rho_{3}}$. It follows from Lemma $2.4(3)$ that $T$ have three positive fixed point $u_{1}, u_{2}, u_{3}$ in $K_{\rho_{1}}^{*}, \Omega_{\rho_{2}} \backslash \bar{K}_{\rho_{1}}^{*}, K_{\rho_{3}}^{*}$ respectively. Therefore, BVP (3.1) have three positive solution $u_{1}, u_{2}, u_{3}$ in $K_{\rho_{1}}^{*}, \Omega_{\rho_{2}} \backslash \bar{K}^{*}{ }_{\rho_{1}}, K_{\rho_{3}}^{*}$ respectively.

Then, BVP (3.1) have three positive solution $u_{1}, u_{2}, u_{3} \in\left[\rho_{1} \varphi(t), \infty\right)$, which means that $u_{1}, u_{2}, u_{3}$ is also the positive solution of BVP (3.1).

Similar to the proof of Theorem 3.1, we can obtain the following Theorems.

Theorem 3.2. Assume that $\left(H_{1}\right),\left(H_{2}\right),\left(H_{3}\right),\left(A_{4}\right)$ hold. Then BVP (1.1) have at least two positive solutions.

Theorem 3.3. Assume that $\left(H_{1}\right),\left(H_{2}\right),\left(H_{3}\right)$ hold and also assume that $\left(A_{1}\right)$ or $\left(A_{2}\right)$ hold. Then BVP (1.1) has at least a positive solution.

\section{Application}

Example 4.1. Consider the following five-point boundary value problem with $p$-Laplacian

$$
\left\{\begin{array}{l}
\left(\phi\left(u^{\prime}\right)\right)^{\prime}+f(t, u)=0, \quad 0<t<1, \\
u^{\prime}(0)=\frac{1}{128} u^{\prime}\left(\frac{1}{4}\right)+\frac{1}{256} u^{\prime}\left(\frac{1}{2}\right)+\frac{1}{64} u^{\prime}\left(\frac{3}{4}\right), \\
u(1)=\frac{1}{8} u\left(\frac{1}{4}\right)-\frac{1}{64} u\left(\frac{1}{2}\right),
\end{array}\right.
$$


where $a_{1}=\frac{1}{128}, a_{2}=\frac{1}{256}, a_{3}=\frac{1}{64}, b_{1}=\frac{1}{8}, b_{2}=\frac{1}{64}, b_{3}=0, \xi_{1}=\frac{1}{4}, \xi_{2}=\frac{1}{2}, \xi_{3}=\frac{3}{4}$,

$$
\begin{gathered}
\phi(u)=\left\{\begin{array}{cl}
-u^{2}, & u \leq 0, \\
u^{2}, & u>0,
\end{array}\right. \\
f(t, u)=\left\{\begin{array}{cc}
\frac{1}{5}(1+t)\left(u(t)-\frac{\varphi(t)}{2}\right)^{30}, & (t, u) \in[0,1] \times(0,2], \\
\frac{1}{5}(1+t)\left(2-\frac{\varphi(t)}{2}\right)^{30}, & (t, u) \in[0,1] \times(2,+\infty) .
\end{array}\right.
\end{gathered}
$$

It is easy to check that $f:[0,1] \times[0,+\infty) \longrightarrow[0,+\infty)$ is continuous. It follows from a direct calculation that

$$
\begin{gathered}
m=\left\{\frac{\left(1+\sum_{i=k+1}^{s} b_{i}+\sum_{i=s+1}^{m-2} b_{i}\right) \phi^{-1}\left((l+1) \int_{0}^{1} a(s) \mathrm{d} s\right)}{1-\sum_{i=1}^{k} b_{i}+\sum_{i=k+1}^{s} b_{i}}\right\}^{-1}=0.96 \\
\gamma=\frac{\left(\sum_{i=1}^{k} b_{i}-\sum_{i=k+1}^{s} b_{i}\right)\left(1-\xi_{k}\right)}{1-\sum_{i=1}^{k} b_{i} \xi_{k}+\sum_{i=k+1}^{s} b_{i} \xi_{k}}=\frac{21}{250} . \\
M=\left\{\frac{\sum_{i=1}^{k} b_{i}-\sum_{i=k+1}^{s} b_{i}}{1-\sum_{i=1}^{k} b_{i}+\sum_{i=k+1}^{s} b_{i}} \int_{\xi_{k}}^{1} \phi^{-1}\left(\int_{0}^{s} a(r) \mathrm{d} r\right) \mathrm{d} s\right\} \\
\end{gathered}
$$

Choose $\rho_{1}=1, \rho_{2}=250$, it is easy to check that $\rho_{1}<\gamma \rho_{2}$, and

$$
\begin{gathered}
f(t, u)>0, t \in[0,1], u \in[\varphi(t),+\infty) ; \\
f_{\rho_{1} \varphi(t)}^{\rho_{1}}=\max \left\{\max _{0 \leq t \leq 1} \frac{\frac{1}{5}(1+t)\left(u(t)-\frac{\varphi(t)}{2}\right)^{30}}{1^{2}}\right\}=\frac{\frac{1}{5}(1+1) 1^{30}}{1^{2}}=\frac{2}{5} \\
<\phi(m)=m^{2}=0.92, t \in[0,1], u \in\left[\varphi(t) \rho_{1}, \rho_{1}\right] ; \\
f_{\gamma \rho_{2}}^{\rho_{2}}=\min \left\{\min _{\frac{3}{4} \leq t \leq 1} \frac{\frac{1}{5}(1+t)\left(2-\frac{\varphi(t)}{2}\right)^{30}}{250^{2}}\right\}=\frac{\frac{1}{5}\left(1+\frac{3}{4}\right)\left(2-\frac{1}{2}\right)^{30}}{250^{2}}=1.074 \\
>\phi(M \gamma)=(M \gamma)^{2}=0.004, t \in\left[\frac{3}{4}, 1\right], u \in\left[\gamma \rho_{2}, \rho_{2}\right] .
\end{gathered}
$$

It follows that $f$ satisfies the conditions $\left(A_{1}\right)$ of Theorem 3.3 , then problems 1.1 and (2.1) have at least two positive solutions.

Remark 4.2. Let $\varphi(u)=u$, the problem is second order $m$-point boundary value problem.

Remark 4.3. Let $\phi_{p}(s)=|s|^{p-2} s, p>1$, the problem is boundary value problem with $p$-Laplacian operators.

\section{Acknowledgements}

The authors are supported by National Natural Science Foundation of China(11371221), the MOE Layout Foundation of Humanities and Social Sciences (13YJAZH091) and National Social Science Fund Project (15BJY183). 


\section{References}

[1] D. J. Guo, V. Lakshmikantham, Nonlinear Problems in Abstract Cone, Academic Press, Inc. Boston, (1988). 2.4

[2] C. P. Gupta, Solvability of a three-point nonlinear boundary value problem for a second order ordinary differential equation, J. Math. Anal. Appl., 168 (1992), 540-551. 1

[3] V. A. Il'in, E. I. Moiseev, Nonlocal boundary value problem of the second kind for a Sturm-Liouville operator, Differ. Equ., 23 (1987), 979-987. 1

[4] V. A. Il'in, E. I. Moiseev, Nonlocal boundary value problem of the first kind for a Sturm-Liouville operator in its differential and finite difference aspects, Differ. Equ., 23 (1987), 803-810. 1

[5] R. Ma, positive solutions for a nonlinear m-point boundary value problems, Comput. Math. Appl., 42 (2001), 755-765. 1

[6] D. X. Ma, Z. J. Du ,W. G. Ge, Existence and iteration of monotone positive solutions for multipoint boundary value problems with p-Laplacian operator, Comput. Math. Appl., 50 (2005), 729-739. 1

[7] M. Moshinsky, Sobre los problems de condiciones a la frontiera en una dimension, Bol. Soc. Math. Mexicana, 7 (1950), 1-25. 1, 2, 2.5

[8] H. Su, Positive Solutions for n-order m-point p-Laplacian Operator Singular Boundary Value Problems, Appl. Math. Comput., 199 (2008), 122-132. 1

[9] H. Su, B. Wang, Z. Wei, X. Zhang, Positive Solutions of four-Point Boundary Value Problems for Higher-order p-Laplacian operator, J. Math. Anal. Appl., 330 (2007), 836-851. 1

[10] H. Su, Z. Wei, B. Wang, The existence of positive solutions for a nonlinear four-point singular boundary value problems with a p-Laplacian operator, Nonlinear Anal., 66 (2007), 2204-2217. 1

[11] H. Su, Z. Wei, F. Xu, The existence of positive solutions for nonlinear singular boundary value system with p-Laplacian, J. Appl. Math. Comp., 325 (2007), 826-836. 1

[12] H. Su, Z. Wei, F. Xu, The wxistence of countably many positive solutions for a system of nonlinear singular boundary value problems with The p-Laplacian operator, J. Math. Anal. Appl., 325 (2007), 319-332. 1.

[13] S. Timoshenko, Theory of Elastic Stability, McGraw-Hill, New York, (1961). 1

[14] Y. Wang, C. Hou, Existence of multiple positive solutions for one-dimensional p-Laplacian, J. Math.Anal.Appl., 315 (2006), 144-153. 1

[15] F. Xu, Positive solutions for multipoint boundary value problems with one-dimensional p-Laplacian operator, J. Appl. Math. Comput., 194 (2007), 366-380. 1, 2.2, 2

[16] X. Zhang, Positive solutions for three-point semipositone boundary value problems with convex nonlinearity, J. Appl. Math. Comp., 30 (2009), 349-367. 1

[17] X. Zhang, L. Liu, Positive solutions for m-point boundary-value problems with one-dimensional p-Laplacian, J. Appl. Math. Comp., 37 (2011), 523-531. 1

[18] X. Zhang, L. Liu, Y. Wu, The eigenvalue problem for a singular higher fractional differential equation involving fractional derivatives, Appl. Math. Comput., 218 (2012), 8526-8536. 1

[19] X. Zhang, L. Liu, Y. Wu, The uniqueness of positive solution for a singular fractional differential system involving derivatives, Commun. Nonlinear Sci. Numer. Simul., 18 (2013), 1400-1409. 1

[20] X. Zhang, L. Liu, Y. Wu, B. Wiwatanapataphee, The spectral analysis for a singular fractional differential equation with a signed measure, Appl. Math. Comput., 257 (2015), 252-263. 1 